\title{
Experimental Validation of an Anti-Lock Braking System for Snowmobiles with Lateral Stability Considerations
}

\author{
M. Corno, F. Roselli, L. Onesto, F. Molinaro, E. Graves, A. Doubek and S.M. Savaresi
}

\begin{abstract}
Anti-lock braking systems are one of the most important safety systems for wheeled vehicles. They reduce the braking distance and, most importantly, help the user maintain controllability and steerability of the vehicle. This paper extends and adapts the concept of Anti-lock braking systems to tracked vehicles, in particular to snowmobiles. Snowmobiles are an interesting development platform for two main reasons: 1) track dynamics, despite being analogous to tyre dynamics, present important differences that help understanding the features of the control algorithm and 2) snowmobiles are simple and rugged vehicles with a limited set of sensors, making the design of an effective control system challenging. The paper designs a trackdeceleration based ABS algorithm and tests it both in straight riding and cornering. The analysis shows that, on snowmobiles, Anti-lock braking systems have negligible advantages in term of stopping distance, but are beneficial in terms of steerability and stability, especially during cornering.
\end{abstract}

\section{INTRODUCTION AND MOTIVATION}

Anti-lock braking systems (ABS) are arguably the most important active safety system for wheeled vehicles. ABS's, by modulating the braking pressure, prevent the wheels from locking and consequently optimize tire forces. During a well modulate ABS braking maneuver, the system makes sure that the tires have enough lateral force to maintain steerability and stability. In fact, when the wheels lock, they rapidly loose the ability to exert lateral forces.

ABS's for wheeled vehicles have received a large amount of attention. See [24] for an overview of the main issues. The literature investigates several actuators [15], [21], [26], sensor configurations [1], [16], [19] and control algorithms including sliding mode control [8], Linear-Parameter varying control [5], [14], fuzzy control [20], switched control [3], and direct control methods [9].

The most advanced (and best performing) techniques are based on control of the tire slip, the normalized difference between the wheel rotational speed and the vehicle speed [22]. However, these algorithms require an accurate vehicle speed estimate. Estimation techniques exist that require a number of additional sensors: GPS, accelerometers, multiple wheel measurements.

Corno, Roselli, Onesto and Savaresi are with the Dipartimento di Elettronica, Informazione e Bioingegneria, Politecnico di Milano, Piazza Leonardo da Vinci 32, 20133 Milano, Italy. sergio.savaresi@polimi.it, matteo.corno@polimi.it, luca.onesto@polimi.it, federico.roselliepolimi.it, Molinaro, Graves and Doubek are with Hayes Performance Systems, Mequon, WI, USA: frank.molinaroahbpsi.com, erik.graves@hbpsi.com, andrew. doubek@hbpsi . com. Corresponding author: Corno

This research was supported by HAYES Performance Systems, Mequon, WI, USA.
The deceleration-based approaches adopt another philosophy, being based mainly on wheel deceleration, they do not heavily rely on the actual vehicle speed. As a result, they deliver worse performance but are more robust [10], [23] and are more easily applicable to low-cost vehicles which cannot rely on a full sensor suite.

Recreational and low-cost vehicles have not received the same amount of attention as passenger cars. This is due to several factors; among the most relevant ones: a smaller market, and the fact that, in most cases, solutions developed for passenger cars are also applicable to low-cost vehicles. Yet in some specific cases, recreational and low-cost vehicles pose challenges that are of a different nature than the corresponding problems in passenger vehicles.

Snowmobiles are an example of a vehicle class that has not received much attention in the literature. Snowmobiles are recreational and utility vehicles, which despite their small overall numbers are very common in some region of the world. In 2016, a total of over 120.000 sleds were sold.

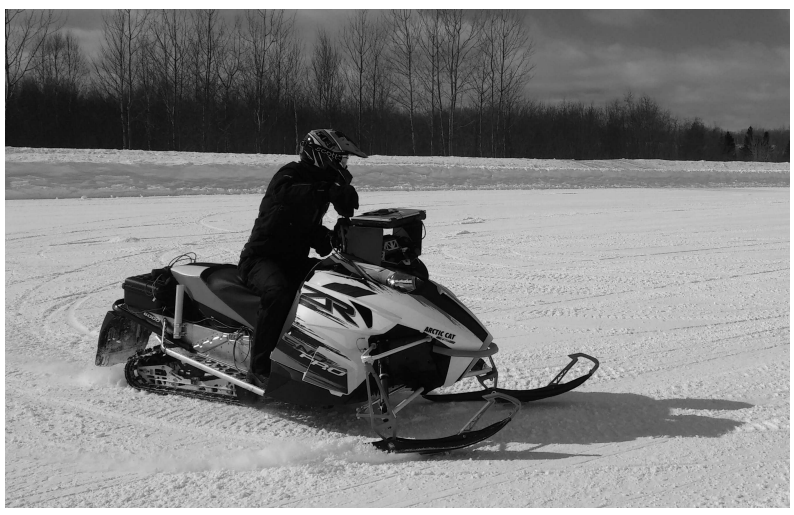

Fig. 1. The trail snowmobile employed in this work.

Snowmobiles (see Figure 1) are a unique class of tracked vehicles, being steered by front skies instead of the more common differential drive. These dynamics, along with the fact that the snow conditions may suddenly vary, makes snowmobiling challenging. An advanced active stability control approach may increase safety without impacting riding thrill; in a similar way to what happened to sport motorcycles [4].

The open scientific and technical literature on snowmobiles is rather scarce. Most of the work has been carried out on engine modeling and emission reduction. The vehicle dynamics are almost completely unexplored and only a handful of works focus on braking dynamics. The literature adopts the accident reconstruction point of view [6], [7], rather than the accident 
prevention. In particular [17] studies the braking characteristics of a recreational snowmobile concluding that "It was determined further that the most effective braking was achieved when the track was locked"; in their conclusions, the authors do not address the coupling between lateral and longitudinal dynamics. Warner and co-authors [27] more recently studied the cornering and acceleration dynamics of a snowmobile, but once again the lateral-longitudinal dynamic coupling, so well explored in the wheeled-vehicle field, is not discussed. To the best of the author's knowledge, active vehicle dynamics control systems for snowmobiles are completely unexplored.

This paper develops a track-deceleration-based ABS algorithm, discussing details of the main features of the algorithm and how they address the peculiarities encountered in the specific application. In designing the algorithm, one has to account for mainly three specific factors: 1) snowmobiles have a very reduced set of sensors 2) the track inertia is considerably higher than a wheel inertia 3) the snow-track friction characteristic exhibits differences with respect to the roadtyre case. These considerations are the basis for the design of the algorithm. The designed algorithm is thoroughly tested and validated on an instrumented vehicle. The experimental protocol explores both the longitudinal aspects of braking and the coupled longitudinal-lateral dynamics.

The papers offers a number of new contributions 1) a previously unpublished analysis of the braking dynamics of tracked vehicles on snow 2) an adaptation of the hybrid ABS algorithm with time varying thresholds 3) a vehicle velocity estimation based only on the track speed and 4) a thorough validation of the hybrid ABS algorithm (in the cited literature the algorithm has only been tested in simulation or on a roller test bench with extremely accurate and fast pressure control).

The paper is organized as follows: Section II describes the experimental setup and provides a simulation model. Section III describes the main aspects of the algorithm and its tuning. Section IV extensively tests the algorithm in two main conditions: straight line braking and cornering braking. Finally, Section V draws some conclusions.

\section{System Modeling}

The present work refers to a modified Arctic Cat ZR 6000 RS trail snowmobile (see Figure 1). The modifications include

- A single-channel Hydraulically Actuated Brake (HAB) manufactured by BWI Group.

- A track speed encoder that measures the track rotational speed. In what follows, the track rotation speed $\omega_{\text {track }}$ is converted into an equivalent track longitudinal speed $\left(v_{\text {track }}\right)$ via

$$
v_{\text {track }}=\frac{L_{\text {track }}}{2 \pi} \omega_{\text {track }}
$$

- A 6 DoF Inertial Measurement Unit (IMU). The data collected by the IMU is only employed for data analysis and not for closed loop control.
- A Racelogic VBOX unit with a GPS antenna to measure the actual vehicle velocity. The data collected by the VOBX is only employed for data analysis and not for closed loop control.

- Two Honeywell pressure transducers: master cylinder pressure and caliper pressure. They are only employed for data analysis and model validation but not for closed loop control.

- A string pot steering angle sensor, only employed for data analysis and not for closed loop control.

- A rapid control system prototyping unit that collects the available sensor inputs and controls the HAB.

The braking system (pads, lever and Master Cylinder (MC)) are standard production grade.

The single-channel HAB enables a limited level of controllability [24]. Figure 2 shows the basic structure of the braking system. It has three control variables: 1) a two-state build

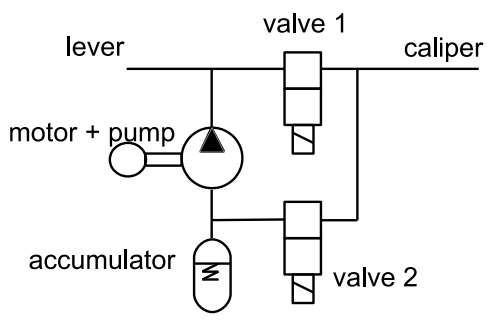

Fig. 2. Hyrdraulic Schematic of the Single-Channel HAB installed on the snowmobile.

valve (valve 1), 2) a two-state release valve (valve 2) and 3) a Brushed DC motor. The brushed DC motor is simply used to empty the accumulator. Based on the state of the two valves three different configurations exist:

- build valve open, release valve closed. The HAB is inactive, the rider controls the caliper pressure by modulating the brake lever. This state is usually referred to as increase.

- build valve closed, release valve open. The HAB is active, the caliper pressure drops. This state is usually referred to as decrease.

- build valve closed, release valve closed. The HAB is active, the caliper holds the pressure. This state is usually referred to as hold.

Note that the pressure increase and decrease rates are determined by the rider force on the lever and the pressure in the caliper. This is not a modulating brake.

The tuning and analysis of the controller is simplified by the availability of a model. The proposed model is composed of two main subsystems: the HAB model and the sled model. The developed HAB model is a physics based model that accounts for the fluid compressibility and the valve flow characteristics; see [11], [24] for more details on the modeling of such systems. The model inputs are the valve states and the master cylinder pressure. It is a physics based system whose parameters: hoses sizes, motor characteristics and pump 
volumes are directly measured or known from component datasheets.

Figure 3 plots the comparison between measured and simulated pressure using data taken from a braking maneuver when the ABS activated. The model is fed with the measured master cylinder pressure (i.e. the rider's input) and the commands to the valve recorded during the experiment. The figure shows that the model correctly captures the caliper pressure rise and drop times as well as the pressure ringing; although in an underdamped fashion.
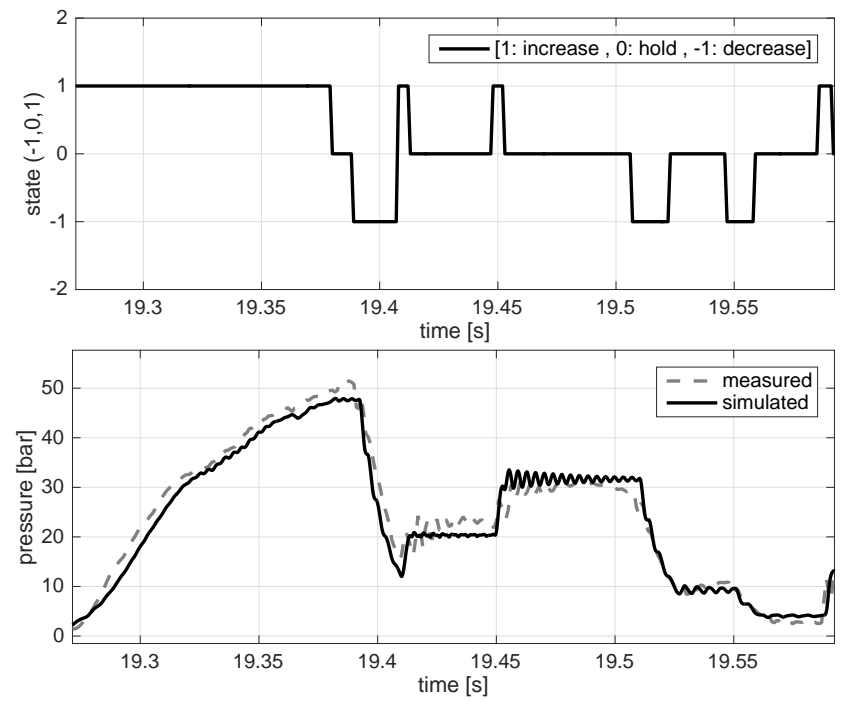

Fig. 3. Validation of the HAB model: valve states (upper plot) and caliper pressure (lower plot)

In modeling the sled, a control-oriented model is adopted. The main governing equations are:

$$
\begin{aligned}
m \dot{v}_{\text {sled }} & =-m g \mu(\lambda) ; \\
J_{\text {track }} \dot{\omega}_{\text {track }} & =-T_{b}+\frac{L_{\text {track }}}{2 \pi} m g \mu(\lambda) ;
\end{aligned}
$$

where $m$ is the sled and rider mass $(580 \mathrm{~kg}), g$ is the gravity acceleration, $v_{\text {sled }}$ is the longitudinal velocity of the vehicle, $J_{\text {track }}$ is the track inertia, $T_{b}$ the braking torque and

$$
\lambda=\frac{v_{\text {track }}-v_{\text {sled }}}{v_{\text {sled }}}
$$

is the equivalent track slip ratio. The most important aspect of the model is the friction force between the track and the snow. Multi-body models of tracked-vehicles, with precise, physics-based models of the track force, are available. The main drawback of those models is that their parameters are difficult to estimate. This work, adopting a control-oriented, single-corner model, avoids the complexity of deriving these models. The model assumes that track force depends only on the equivalent track slip ratio through a nonlinear function $\mu(\lambda)$ which is identified from data.

The repetition of a number of open-loop manual braking maneuvers at different deceleration levels reveals the relation between the equivalent track slip and the snow-track friction coefficient. Figure 4 plots the results in terms of $\lambda$ and $\mu$. In figure, the track slip has been measured by the Racelogic

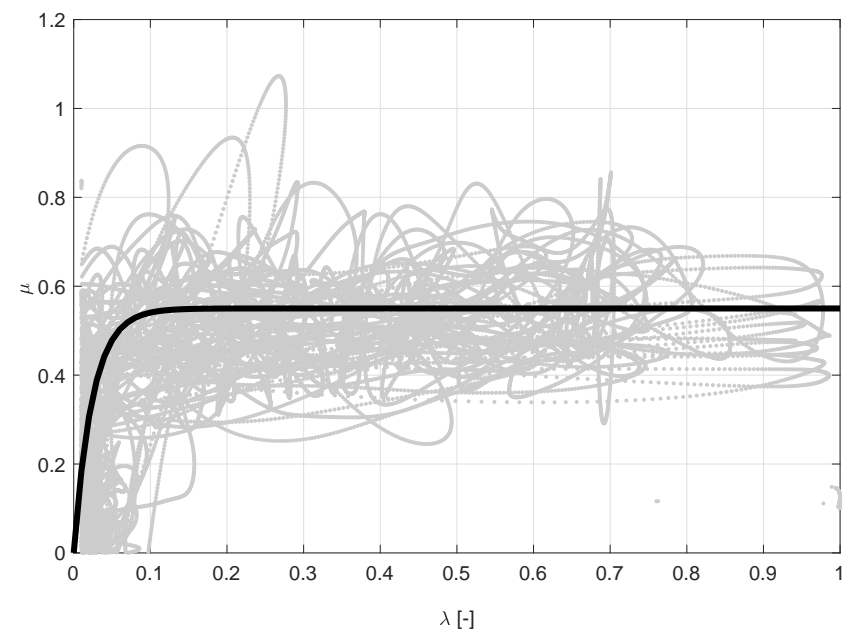

Fig. 4. Track force characteristics measured data and fitted Burkhardt model.

VBOX and the track encoder. The longitudinal acceleration reading comes from the IMU. $\mu$ is obtained by an unfiltered $a_{x}$ and the knowledge of the mass. These experimental data can be used to fit a model; the Burkhardt formula [2] satisfactorily fits the snow-track friction behavior. The fitting is particularly good for track slip values below $50 \%$ where the ABS will operate.

The data reveal a force characteristic with a relatively steep increase of force at low track slip and a flat characteristic for high slip. Once the track characteristic is known, a parametric identification can be run to identify the two remaining model parameters: the brake friction coefficient (relating braking pressure and braking torque) and the equivalent track inertia (estimated at $5 \mathrm{kgm}^{2}$ ). Figure 5 shows the sled model val-
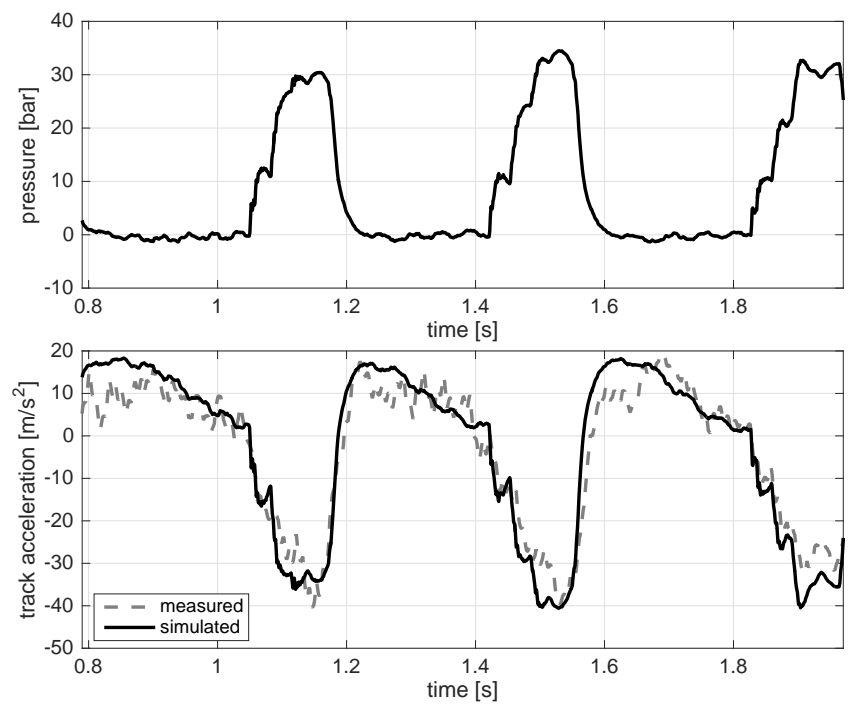

Fig. 5. Time-domain validation of the sled model: braking pressure (upper plot) and track acceleration (lower plot).

idation during a simplified ABS cycle. As it can be seen, 
the model describes the track deceleration within a $5 \mathrm{~m} / \mathrm{s}^{2}$ instantaneous error.

\section{Control System Design}

The model analysis shows that track and tyre dynamics can be accurately described by similar models (see (1)-(2)); this justifies the choice to base the design of the ABS algorithm on the solutions already developed for wheeled vehicles [3], [10], [23]. The core of the algorithm is shown in Figure 6. The algorithm main input is the equivalent track acceleration

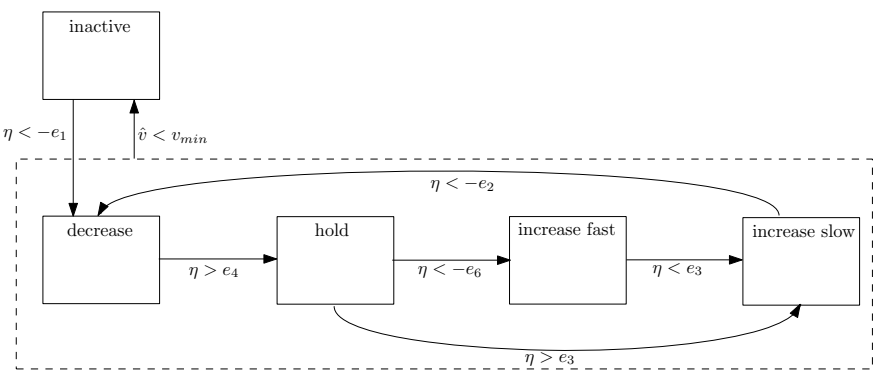

Fig. 6. Finite State Machine describing the core of the algorithm.

$\eta=\dot{v}_{\text {track }}$. In summary, the main idea is to compare $\eta$ against thresholds that represent the maximum deceleration/acceleration that the vehicle can physically achieve. If the track is decelerating more than what the friction permits, it means that the track has broken the friction limits and it is slipping. The ABS algorithm detects these moments and commands a decrease of the braking pressure. This causes the slip to decrease; the track velocity will re-align with the vehicle velocity, determining a track acceleration. The track acceleration can be employed to detect when the slip has decreased too much; at this point, the pressure needs to be increased to guarantee a braking action. The algorithm has two increase states: an increase slow and increase fast, in which the rate of change of the pressure is different. The two different rates of increase of braking pressure are obtained by actuating the valves in a Pulse Width Modulation (PWM) fashion, with two different Duty Cycles.

The proposed algorithm implements the idea of [23] with a number of variations. The differences are mainly due to the fact that the original algorithm relies on a modulating braking torque control, whereas the present vehicle is equipped with a single channel HAB. More in details, the differences are:

- The original algorithm has 5 phases, more precisely, it has a hold phase also after the increase phase. Our implementation skips that phase. The reasons for this choice are two-fold: in off-road applications, rider prefers limit cycles that venture more in the high slip region; and, because the track force characteristic is predominantly flat (see Figure 4), the condition for existence and stability of the limit cycles derived in [23] could not be met with the original algorithm.

- The original algorithm does not provide an exit condition. Our implementation exits from the control algorithm when the vehicle estimated velocity drops below $v_{\text {min }}$. The next subsection describes the vehicle velocity estimation algorithm in more details. This is required by the fact that as the vehicle velocity decreases, the slip dynamics get infinitely fast [24] and this makes it impossible for the control algorithm to perform at low velocity.

- To account for the dependency of the slip dynamics on the velocity, some of the thresholds $\left(e_{1}\right.$ and $\left.e_{2}\right)$ are scheduled with the estimated velocity. In particular, $e_{1}$ and $e_{2}$ are monotonically increasing with $\hat{v}$. Note that the original algorithm accounted for this velocity dependency by scheduling the rate of change of the pressure; this solution is not pursuable with the available HAB.

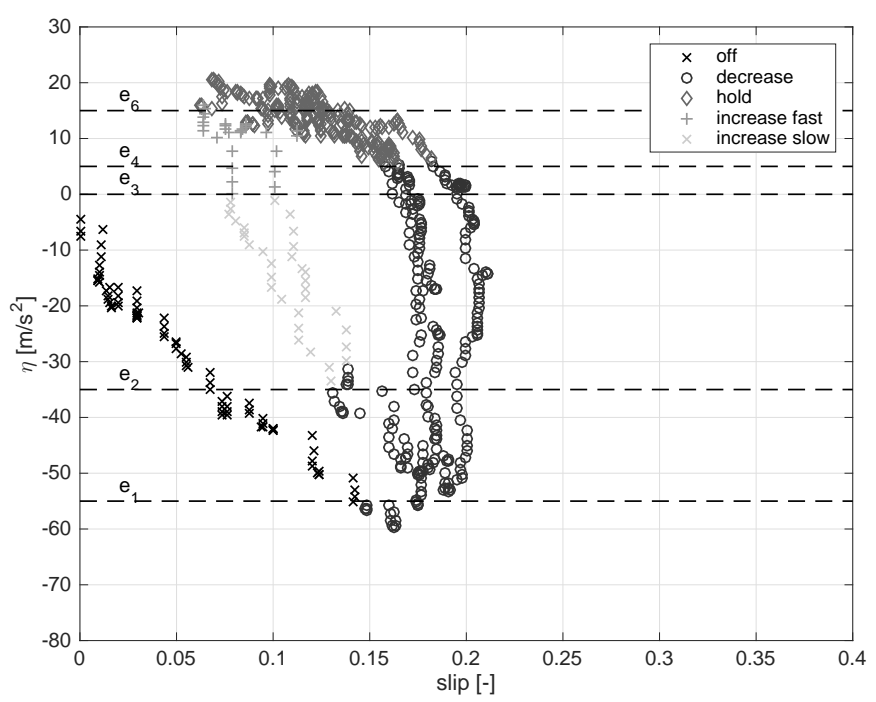

Fig. 7. Phase portrait of a section of a panic braking maneuver on the instrumented snowmobile.

Figure 7 plots three iterations of the limit cycle as recorded during a test brake on the snowmobile. The plot represents the results in the phase plane of the two state variables, slip and wheel normalized acceleration. Different symbols indicate the different phases of the algorithm. The following remarks are due:

- The PWM modulation of the pressure during the two increase phases is effective in obtaining two different dynamics. In the increase fast phase - which is considerably shorter - the system trajectory is considerably more vertical than in the increase slow phase.

- The phase portrait exhibits some nonidealities with respect to the simulated ones in [23], these are due to the different type of actuators employed. The original work assumed a precise control of the braking torque; the proposed algorithm, on the other hand, deals with actual, real world actuators and measurements.

- The ABS successfully control the track slip, which oscillates around 0.15 . 


\section{A. Vehicle velocity estimation}

The ABS algorithm aims at controlling the track slip. The proposed algorithm does that indirectly using the track acceleration; yet it needs an estimate of the the vehicle speed to schedule thresholds $e_{1}$ and $e_{2}$ and to turn off the algorithm. Vehicle velocity estimation is a well known problem in the automotive field with a number of interesting approaches [12], [13], [18], [25]. Available solutions tend to rely on sensors normally available on cars: four wheel rotational speed, an inertial platform units and in some cases GPS. The sensor set available on snowmobiles is considerably smaller: only the track speed. The estimation accuracy suffers from this limitation, yet the knowledge of the ABS algorithm can be incorporated in the estimation.

When the ABS is cycling, the track slip oscillates between a minimum and maximum value: the minimum slip points are the points where the track speed is the closest to the chassis speed. The velocity estimation rationale is thus to exploit the measure of the track speed at these instants; these are known as they are synched with the ABS phases. In particular, at the beginning of the increase phase the track slip is going to be at its minimum. Despite this, if the ABS is cycling at high track slip, the velocity at these time instants could be imprecise. This effect can be minimized by estimating the average track deceleration and integrating that value over the ABS cycles. In the estimate update phase, triggered at the same time as the ABS increase phase, the average track deceleration $a_{x, a v g}$ is computed as the difference between the track velocity when the phase is triggered and the track velocity of the previous estimation algorithm estimation. The average acceleration is then integrated. The resulting algorithm is shown in Figure 8. The proposed approach has some advantages:

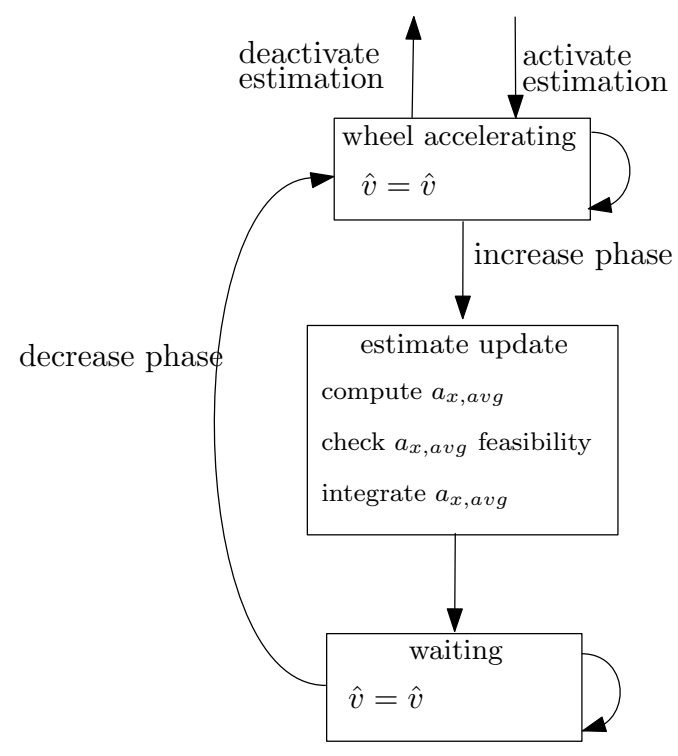

Fig. 8. Finite State Machine describing the velocity estimation algorithm.

- since the estimated velocity is not used for ABS activation the estimation triggering threshold can be tuned less conservatively than the deceleration that activates the
ABS. In this way, even if in the first ABS cycle the track slip reaches very high values, the estimation is not affected.

- A second advantage of working with an integral approach is that one can incorporate additional information on the maximum deceleration that the vehicle can reach: if the track-based estimation of the deceleration is above a prespecified threshold, the average deceleration is saturated to the maximum possible deceleration (in this work a maximum deceleration of $0.6 \mathrm{~g}$ is considered).

- Should a longitudinal body accelerometer become available on higher-end vehicles; the measure can be easily incorporated in the approach by substituting the average deceleration computed from the track speed, with the inertial one.

Being an integral approach, based solely on the track speed, it is impossible to guarantee that the estimation is accurate in all conditions. For this reason, it can be used only for deactivation and scheduling purposes, i.e. non safety critical functionality. Figure 9 plots two experimental runs with the proposed algorithm; the figure shows the track speed, the estimated velocity and the actual velocity measured with the GPS system, in the best and worst case scenarios. In the best
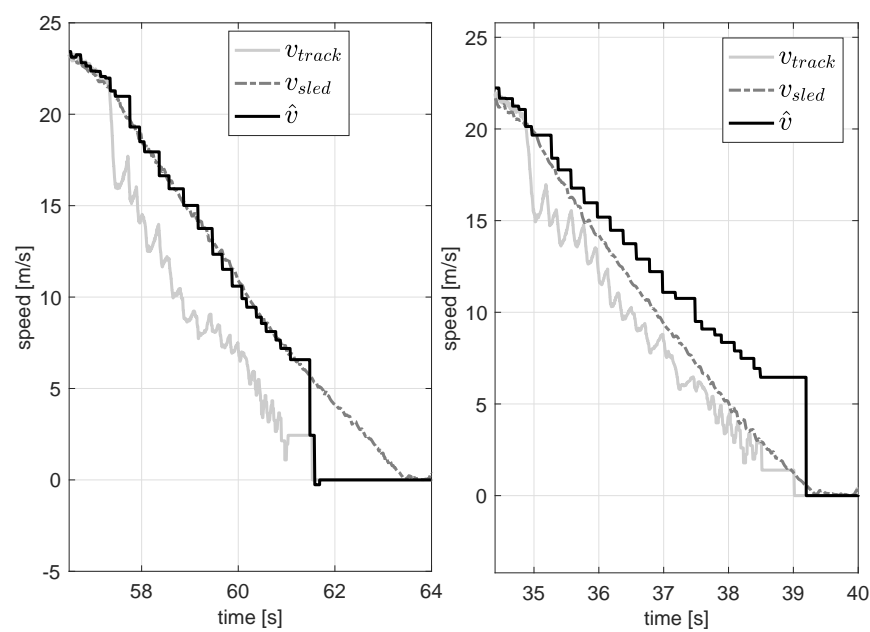

Fig. 9. Vehicle velocity estimation algorithm results for two braking maneuvers. The figure compare the estimation against the track speed and the actual estimated speed measure by the Racelogic VBOX.

scenario, the algorithm provides a very accurate estimate of the vehicle speed; in the worst case scenario, the velocity is overestimated because of integral drift. Nevertheless, it is sufficiently accurate for scheduling purposes.

\section{EXPERIMENTAL VALIDATION}

This section quantitatively assesses the performance of the ABS system in two scenarios: straight line braking and cornering performance. All tests have been performed at the test course of the Keweenaw Research Center, a research institute of Michigan Technological University. The snowmobile is ridden on packed groomed snow, the rider was instructed to 
perform each braking maneuver on a different area of the track, to guarantee repeatability. The rider is one of the authors with several year of riding experience.

\section{A. Straight line performance}

The ABS system is designed and tuned for straight riding. The protocol consists in panic braking from different initial speeds (ranging from 10 to $24 \mathrm{~m} / \mathrm{s}$ ) and with the rider acting on the steering to maintain the snowmobile's trajectory. The rider performed a total of 20 maneuvers with the final tuning discussed thereafter (the calibration phase is not discussed for space limitation). Figure 10 plots an example of a straight line maneuver. The following remarks are due:
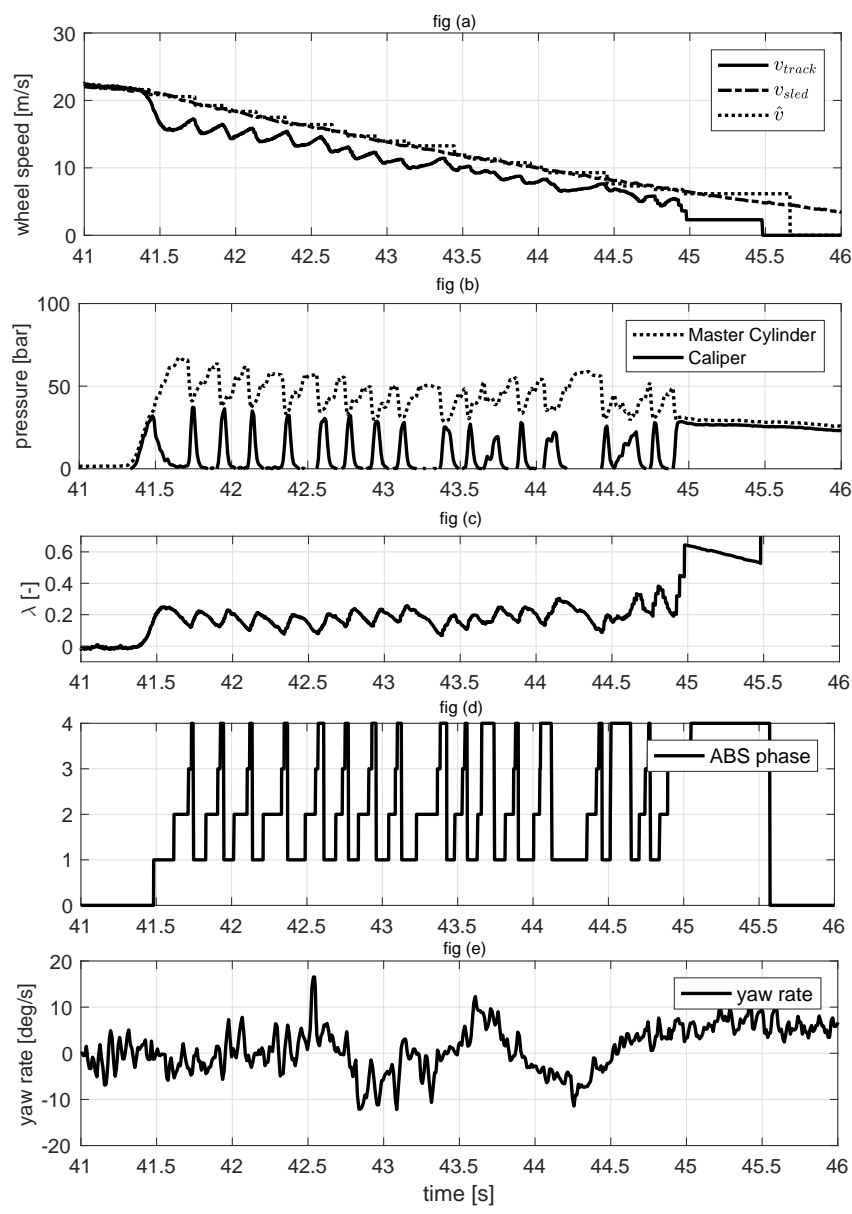

Fig. 10. Time-domain validation of the ABS during a straight panic brake From top to bottom: fig (a): speed, fig (b): braking pressure, fig (c): track slip, fig (d): ABS phase and fig (e): yaw rate.

- The control algorithm maintains the track slip around a value of 0.2 , yielding optimal longitudinal force without excessive track slip.

- During the release phase the caliper pressures reaches 0 due to the large track inertia and the relatively low surface friction force. This essentially means that the hold phase is triggered before intended, yet the algorithm is robust.

- The cycling of the algorithm is stable and repeatable and, thanks to scheduling, only marginally velocity dependent.
- At low speed the track encoder suffers from a strong quantization effect, yet the algorithm is robust.

The above analysis refers to a single run; Figure 11 provides a statistical look a the data. It summarizes the results from three perspectives over repeated tests, assessing the effect of the ABS system with respect to no assistance:

- the mean track slip during the maneuvers;

- the mean deceleration is an indication of the actual braking performance, and is correlated to the stopping distance;

- the yaw rate variance characterizes the vehicle stability during the maneuver.
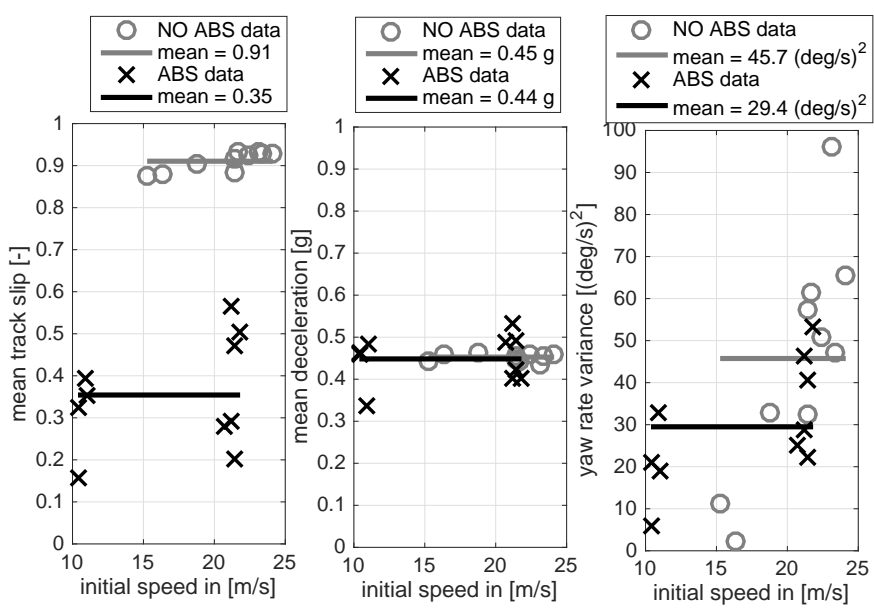

Fig. 11. Summary performance analysis during straight braking: mean track slip (left plot), mean deceleration (center plot), yaw rate variance (right plot).

From Figure 11, one can draw the following conclusions:

- The designed ABS system successfully controls the track slip, maintaining it around a value of 0.35

- Despite the lower track slip, the ABS system does not yield a shorter braking distance. The average deceleration is the same as in the panic brake without ABS control. This confirms the identified track characteristic of Figure 4.

- When the ABS system is active, the yaw rate variance is smaller than in the uncontrolled panic brake. Data indicate a reduction of $35 \%$ in yaw rate variance. This indicates that, during the ABS tests, it was easier for the rider to maintain a straight trajectory. This is consistent with the assumption that the track lateral force drops as the track slip increases. However, no occurrence of loss of controllability occurred during the tests.

In conclusion, when straight riding is considered the ABS system does not seem to considerably decrease the braking distance, but yields slightly more stable stopping events.

\section{B. Cornering performance}

The following tests further investigate the vehicle behavior in combined slip conditions i.e. when the track is engaged both 
in the longitudinal direction and laterally. The rider initiates a steady state corner at constant speed $(12 \mathrm{~m} / \mathrm{s})$ and, once the vehicle is stabilized, brakes as hard as possible. As before, two configurations are tested: in the uncontrolled case, the rider tries to manually stabilize the vehicle and stay on corner by modulating the braking torque and the steering. In the controlled case, the rider pulls the brake lever as hard as possible, he stabilizes the vehicle and stays on corner with the steering. Note that in these conditions, it is not possible to perform the uncontrolled panic brake maneuver because of roll-over risks. Each configuration is tested 10 times.

Figure 12 plots two tests to better appreciate the kind of maneuver: one performed without the ABS, and the latter with the ABS active. From the figure, one can conclude:
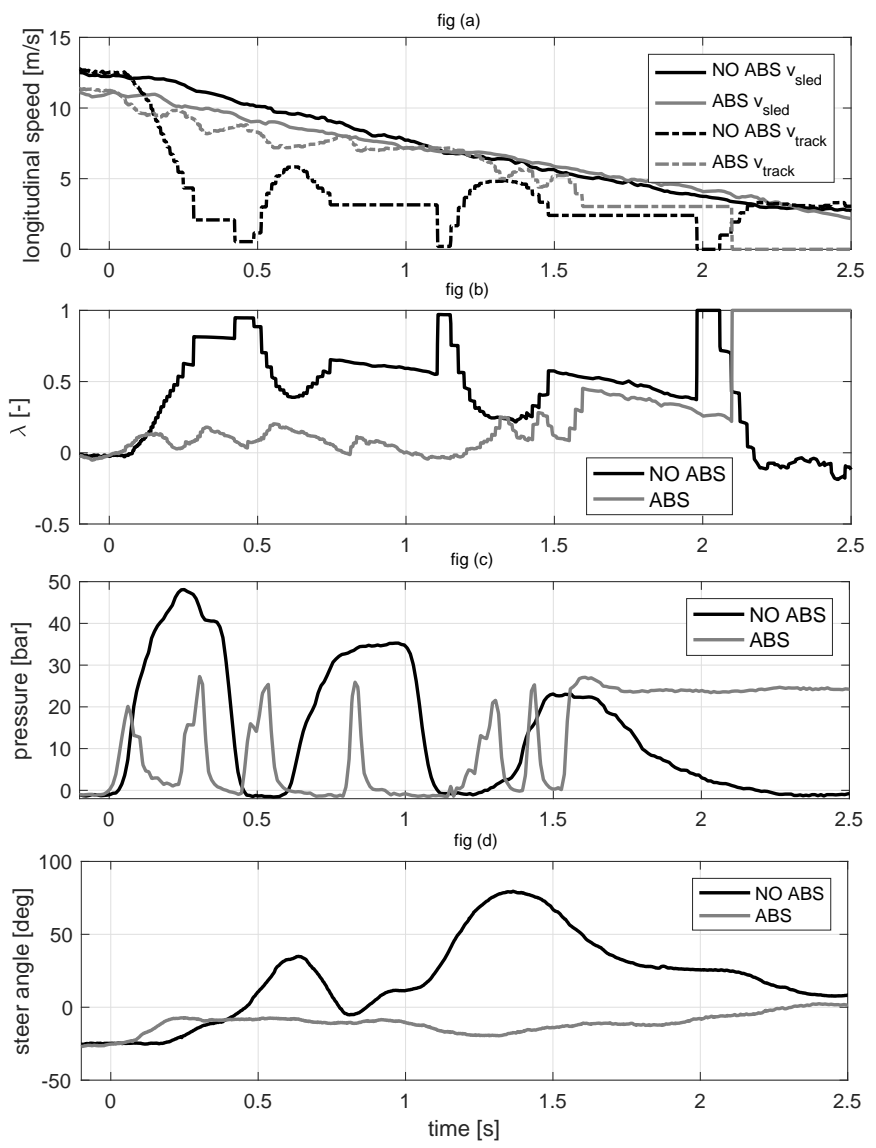

Fig. 12. Time-domain analysis of the brake-in-turn maneuver. fig (a): speed, fig (b): track slip, fig (c) braking pressure and fig (d) steer angle.

- As expected, the ABS system helps to maintain a lower track slip.

- The lower track slip does not translate into a reduced deceleration.

- The rider needs to act on the steering angle much less when the ABS system is active. It is interesting to note that, in order to keep the corner, when the ABS is not active, the rider needs to counter steer.

Figure 13 provides a repeatability and statistical analysis of several braking maneuvers performed in the above conditions.
Four quantitative indexes describe the maneuver in a concise way:

- Average deceleration. It measures the effectiveness of the braking maneuver.

- Average yaw rate. It quantifies how much the trajectory is perturbed by the braking action. The brake lever is pulled at around $20 \%$ s.

- Yaw rate variance. It indicates how stable the trajectory is.

- Average steer. It measures the effect of the braking on the steering characteristics. When going at a constant velocity, there is a constant gain between yaw rate and steering angle that depends on the lateral forces. When the sled brakes, this relation is perturbed.
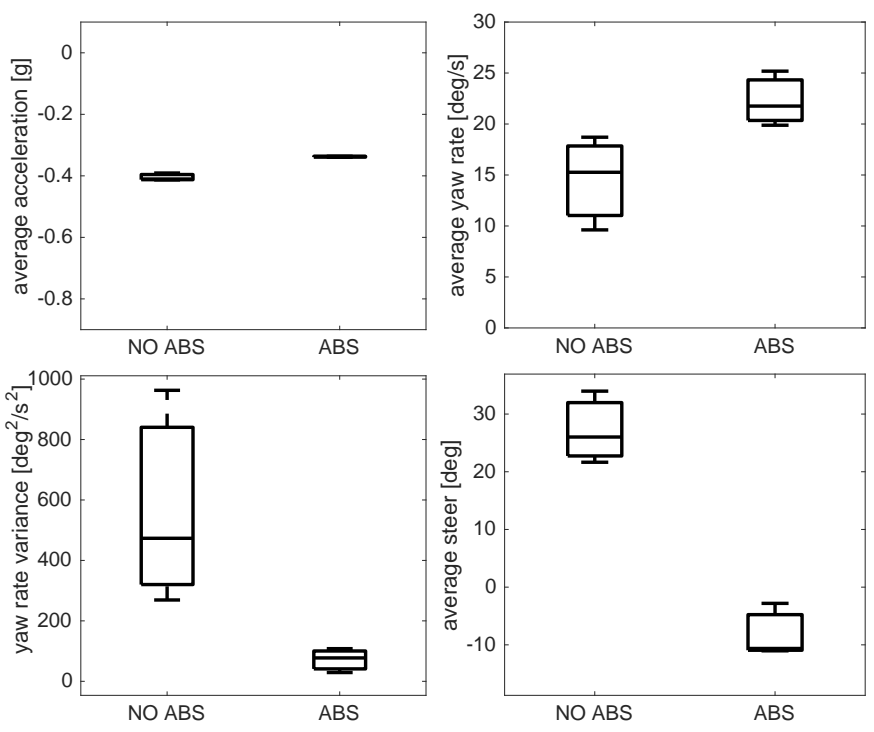

Fig. 13. Brake-in-turn maneuver summary statistics: average acceleration, average yaw rate, yaw rate variance, average steering angle.

The statistical analysis confirms the results of the straight line braking performance; in particular

- The average deceleration is similar in both conditions, with the ABS braking deceleration slightly lower. The reduced spread in terms of longitudinal deceleration is once again given by the rather flat snow-track friction characteristics.

- The ABS yields a higher average yaw rate; the rider finds it easier to track the corner when braking.

- The ease with which the corner is negotiated is also seen in the reduced yaw rate variance; this indicates a more stable corner. The extremely high yaw rate variance is determined by a few maneuvers in which the rider almost lost control of the vehicle. This never happened with the ABS active.

- The steering angle is considerably different. Without ABS the rider heavily compensates with a steering action to the point where a counter-steering maneuver has to be initiated. With the ABS, the rider corrects only of a few 
degrees. Figure 12 shows that the initial steering is around $-25^{\circ}$.

\section{Conclusions}

The paper discusses the adaptation of a hybrid Anti Lock Braking System to a snowmobile. Snowmobiles represent an interesting testbed for mainly two reasons: 1) the track-snow dynamics exhibit some specific features with respect to the tyre-road friction: a higher inertia and a considerably flatter characteristics and 2) snowmobiles rely on an extremely small sensor set (as a matter of fact only the track speed). This calls for the implementation of two ad-hoc specific adaptations of the classical ABS algorithm: velocity dependent thresholds and a signal-based velocity estimation.

The second part of the paper studies the system performance in two different conditions: straight line braking and cornering. The straight line braking serves as a validation of the control system design and modeling. These tests show that the ABS system is capable of limiting the slip during braking; at the same time, the availability of an ABS system confirms the previously available findings ( [17]) and the model showing that track lock up does not impact braking distance negatively. The cornering tests confirm that, although the control system has been designed for straight braking, it performs also during cornering. Furthermore, the cornering tests show that avoiding track lock up is paramount for stable braking during cornering.

\section{REFERENCES}

[1] F Braghin, M Brusarosco, F Cheli, A Cigada, S Manzoni, and F Mancosu. Measurement of contact forces and patch features by means of accelerometers fixed inside the tire to improve future car active control. Vehicle System Dynamics, 44(sup1):3-13, 2006.

[2] Manfred Burckhardt. Fahrwerktechnik: Radschlupf-regelsysteme. 1993. Vogel-Verlag, Wurtzburg.

[3] M. Corno, M. Gerard, M. Verhaegen, and E. Holweg. Hybrid abs control using force measurement. IEEE Transactions on Control Systems Technology, 20(5):1223-1235, 2012.

[4] M. Corno, G. Panzani, and S. M. Savaresi. Single-track vehicle dynamics control: State of the art and perspective. IEEE/ASME TRANSACTIONS ON MECHATRONICS, 20(4):1521-1532, 2015.

[5] M. Corno, Savaresi S.M., and G.J. Balas. On linear parameter varying (LPV) slip-controller design for two-wheeled vehicles. International Journal of Robust and Nonlinear Control, 19(12):1313-1336, 2009.

[6] Pamela D’Addario, Ken Iliadis, and Gunter Siegmund. Predicting snowmobile speed from visible locked-track and rolldown marks in groomed/packed snow conditions. SAE International Journal of Transportation Safety, 4(2016-01-1477):128-133, 2016.

[7] Pamela D'Addario, Kenneth Iliadis, Brian Mac Giolla Ri, and Gunter P Siegmund. Acceleration and braking performance of snowmobiles on groomed/packed snow. SAE International Journal of Passenger CarsMechanical Systems, 4(2011-01-0287):272-278, 2011.

[8] S. Drakunov, U. Özgüner, P. Dix, and B. Ashrafi. ABS control using optimum search via sliding modes. IEEE Transactions on Control Systems Technology, 3(1):79-85, 1995.
[9] Simone Formentin, Pierpaolo De Filippi, Matteo Corno, Mara Tanelli, and Sergio M Savaresi. Data-driven design of braking control systems. IEEE Transactions on Control Systems Technology, 21(1):186-193, 2013.

[10] Mathieu Gerard, William Pasillas-Lepine, Edwin De Vries, and Michel Verhaegen. Improvements to a five-phase abs algorithm for experimental validation. Vehicle System Dynamics, 50(10):1585-1611, 2012.

[11] J Christian Gerdes and JK Hadrick. Brake system modeling for simulation and control. Journal of Dynamic Systems, Measurement, and Control, 121:496-503, 1999.

[12] Havard Fjaer Grip, Lars Imsland, Tor A Johansen, Jens C Kalkkuhl, and Avshalom Suissa. Vehicle sideslip estimation. IEEE Control. Syst. Mag., 29(5):36-52, 2009.

[13] Lars Imsland, Tor A Johansen, Thor I Fossen, HåVard FjæR Grip, Jens C Kalkkuhl, and Avshalom Suissa. Vehicle velocity estimation using nonlinear observers. Automatica, 42(12):2091-2103, 2006.

[14] T.A. Johansen, I. Petersen, J. Kalkkuhl, and J. Lüdemann. Gainscheduled wheel slip control in automotive brake systems. IEEE Transactions on Control Systems Technology, 11(6):799-811, November 2003.

[15] M. Kees, K. J. Burnham, F. P. Lockett, J. H. Tabor, and R. A. Williams. Hydraulic actuated brake and electromechanically actuated brake systems. In 2001 ADAS. International Conference on Advanced Driver Assistance Systems, (IEE Conf. Publ. No. 483), pages 43-47, 2001.

[16] S. Kerst, B. Shyrokau, and E. Holweg. Reconstruction of wheel forces using an intelligent bearing. SAE International Journal of Passenger Cars - Electronic and Electrical Systems, 9(1):196-203, 2016.

[17] James KH Kho and James A Newman. Braking characteristics of the recreational snowmobile. Technical report, SAE Technical Paper, 1973.

[18] Matthijs Klomp, Yunlong Gao, and Fredrik Bruzelius. Longitudinal velocity and road slope estimation in hybrid electric vehicles employing early detection of excessive wheel slip. Veh. Syst. Dynamics, 52(sup1):172-188, 2014.

[19] A. Kunnappillil Madhusudhanan, M. Corno, M.A. Arat, and E. Holweg. Load sensing bearing based road-tyre friction estimation considering combined tyre slip. Mechatronics, 39:136-146, 2016.

[20] C.M. Lin and C.F. Hsu. Self-learning fuzzy sliding-mode control for antilock braking systems. IEEE Transactions on Control Systems Technology, 11(2):273-278, 2002.

[21] H. Park and S. B. Choi. Development of a sensorless control method for a self-energizing brake system using noncircular gears. IEEE Transactions on Control Systems Technology, 21(4):1328-1339, July 2013.

[22] W. Pasillas-Lapine, A. Loria, and M. Gerard. Design and experimental validation of a nonlinear wheel slip control algorithm. Automatica, 48(8):1852-1859, 2012.

[23] W. Pasillas-Lepine. Hybrid modeling and limit cycle analysis for a class of five-phase anti-lock brake algorithms. Vehicle System Dynamics, 44(2):173-188, 2006.

[24] S.M. Savaresi and M. Tanelli. Active Braking Control Design for Road Vehicles. Springer, 2010.

[25] S. Semmler, D. Fischer, R. Isermann, R. Schwarz, and Rieth P. Estimation of vehicle velocity using brake-by-wire actuators. In Proceedings of the 15th IFAC World Congress, Barcelona, Spain, July, 21-26 2002.

[26] Fabio Todeschini, Matteo Corno, Giulio Panzani, and Sergio M Savaresi. Adaptive position-pressure control of a brake by wire actuator for sport motorcycles. European Journal of Control, 20(2):79-86, 2014.

[27] Mark H Warner, Jon E Bready, Wyatt Y Warner, and Alan F Asay. Snowmobile cornering and acceleration data from on-snow testing. Technical report, SAE Technical Paper Number 2015-01-1431, 2015. 